Brain and Cognition 39, 133-146 (1999)

Article ID brcg.1998.1063, available online at http://www.idealibrary.com on IDE $\mathbf{l}^{\circledR}$

\title{
Cross-Modal Priming and Explicit Memory in Patients with Verbal Production Deficits
}

\author{
Tim Curran \\ Department of Psychology, Case Western Reserve University \\ and \\ Daniel L. Schacter and Lissa Galluccio \\ Department of Psychology, Harvard University
}

\begin{abstract}
Implicit memory is often thought to reflect an influence of past experience on perceptual processes, yet priming effects are found when the perceptual format of stimuli changes between study and test episodes. Such cross-modal priming effects have been hypothesized to depend upon stimulus recoding processes whereby a stimulus presented in one modality is converted to other perceptual formats. The present research examined recoding accounts of cross-modal priming by testing patients with verbal production deficits that presumably impair the conversion of visual words into auditory/phonological forms. The patients showed normal priming in a visual stem completion task following visual study (Experiment 1), but showed impairments following auditory study in both implicit (Experiment 2) and explicit (Experiment 3) stem completion. The results are consistent with the hypothesis that verbal production processes contribute to the recoding of visual stimuli and support cross-modal priming. The results also indicate that shared processes contribute to both explicit memory and cross-modal implicit memory. () 1999 Academic Press
\end{abstract}

Implicit memory occurs when behavior is affected by previous experience without intentional, conscious recollection of that experience (Roediger \& McDermott, 1993; Schacter, 1987). Perceptual or data-driven implicit memory tasks are those in which memory is cued by a stimulus that is physically related to the target stimulus. For example, in word-stem completion, participants might study the word TRUCK then their memory would be cued indirectly by asking them to complete three-letter stems including TRU_. Per-

This project was supported by NINDS Grant NS27950 and NIA Grant AG08441. We thank David Caplan for providing referrals and background information on the patients. L.G. is currently a graduate student in the Psychology Department at Rutgers University. Correspondence should be sent to Tim Curran, Department of Psychology, Case Western Reserve University, Cleveland, OH 44106-7123. E-mail: tec3@po.cwru.edu. 
ceptual implicit memory is influenced by the physical overlap between the study and test stimuli. For example, priming elicited by visual test cues is greater when target words were studied in the visual rather than auditory modality (Graf, Shimamura \& Squire, 1985; Jacoby \& Dallas, 1981; Rajaram \& Roediger, 1993; Roediger \& Blaxton, 1987; Schacter \& Graf, 1989). Conversely, words studied in the auditory modality produce greater priming when the test cue is also auditory (Bassili, Smith \& MacLeod, 1989; Jackson \& Morton, 1984). Explicit memory is typically less affected by manipulations of study-test modality.

Though modality changes typically affect implicit memory more than explicit memory, the opposite appears to be true for semantic encoding, which affects explicit memory more than perceptual priming (e.g., Challis \& Brodbeck, 1992; Jacoby \& Dallas, 1981; Roediger, Weldon, Stadler \& Riegler, 1992; Schacter \& Church, 1992). These and related findings have inspired the idea that priming of visual words reflects presemantic processes associated with visual word perception (Bower, 1996; Curran \& Schacter, 1996; Keane, Gabrieli, Fennema, Growdon \& Corkin, 1991; Ratcliff \& McKoon, 1996; Roediger, 1990; Schacter, 1990, 1994; Schacter \& Buckner, 1998; Squire, 1994; Tulving \& Schacter, 1990). The basic hypothesis is that prior study of a visual word facilitates (or biases, Ratcliff \& McKoon, 1996) later perception of the cue (e.g., TRU), so that the previously studied word (TRUCK) is more likely to come to mind.

Though the finding that a change in study-test modality reduces priming is consistent with perceptual accounts, purely modality specific perceptual hypotheses have difficulty explaining the fact that significant priming is typically observed in cross-modality conditions. For example, studying and auditory word should not prime mechanisms of visual word perception, so priming may depend on nonperceptual processes. Because semantic features are shared by visual and auditory words, cross-modal implicit memory could reflect semantic priming (e.g., Bassili et al., 1989). Although the semantic hypothesis seems inconsistent with previously discussed observations that implicit memory is affected little by semantic variables, the idea remains viable because small LOP effects on implicit memory are sometimes observed (Brown \& Mitchell, 1994; Challis \& Brodbeck, 1992; Thapar \& Greene, 1994).

Alternative accounts of cross-modal priming have stemmed from the idea that perceptual processing of a stimulus may not be limited to the nominal presentation modality used by the experimenter. The general hypothesis is that a stimulus presented in one modality can be recoded in terms of other modalities (e.g., Downes et al., 1996; Kirsner, Dunn \& Standen, 1989; McClelland \& Pring, 1991). For example, if TRUCK is studied visually, a person could experience its auditory form through (possibly covert) naming processes. Similarly, the stem TRU could be encoded in terms of its pronunciation. Support for this hypothesis is provided by McClelland and Pring 
(1991), who tested priming in an auditory stem completion task that was preceded by the study of visual words in one of three different encoding conditions. Cross-modal priming was greatest when participants named the visual words during study, weaker when they read the words silently, and weakest when they were engaged with an articulatory suppression task (repeating aloud, "one, two, three ...'”). These results suggest that priming increases with the potential for auditory recoding of the visual words. Similarly, Downes et al. (1996) cite an unpublished study from their laboratory in which articulatory suppression during the test phase of visual stem completion eliminated cross-modal priming.

The present study was designed to examine the recoding account of crossmodal priming by testing four brain-injured patients with verbal production deficits. The patients were selected to have normal perception and language comprehension, together with verbal production deficits that are not attributable to peripheral articulation impairments. Their most prominent problem is an inability to produce the phonological form of a word. Theories of lexical processing often distinguish between input phonology and output phonology (Caplan, 1992; Ellis \& Young, 1988; Hillis \& Caramazza, 1995). Because the patients in our study show speech production deficits that are independent of the input modality (pictures, written words, spoken words), a deficit in output phonology is implicated.

In summary, it has been hypothesized that cross-modal priming effects depend on stimulus recoding. If recoding of visual stimuli into an auditory/ phonological format depends upon output phonology, a recoding hypothesis of cross-modal priming predicts that patients with verbal production deficits should show impaired cross-modal priming. The recoding hypothesis was examined in two experiments using a visual word-stem completion task. Words were studied visually in Experiment 1 and auditorily in Experiment 2. In Experiment 1 we examine whether patients with verbal production deficits show normal priming in within-modal conditions. In Experiment 2 we test the hypothesis that verbal production deficits impair cross-modal priming. Experiment 3 is similar to Experiment 2-auditory study followed by visual stem completion-but participants were explicitly instructed to complete the stems with previously studied words.

\section{EXPERIMENT 1}

\section{Method}

Participants. Participants were four brain-injured patients with verbal production deficits. The patients were recruited through Massachusetts General Hospital and paid \$7 per hour for participation. R.C. is a 60-year-old male (at time of testing) with 6 years of formal education who participated in Experiment 13 months after a CVA. He has an extensive left-hemisphere lesion including frontal, parietal, and temporal cortex along with subcortical 
TABLE 1

Proportion Correct on the Psycholinguistic Assessment of Language (PAL)

\begin{tabular}{|c|c|c|c|c|c|c|}
\hline & \multicolumn{2}{|c|}{ Controls } & \multicolumn{4}{|c|}{ Patients } \\
\hline & Mean & $\mathrm{SD}$ & R.C. & L.L. & T.R. & G.Z. \\
\hline \multicolumn{7}{|l|}{ Verbal production tasks } \\
\hline Reading & 1.00 & .01 & $.56^{* *}$ & $.44 * *$ & $.41 * *$ & $.56 * *$ \\
\hline Repetition & .97 & .04 & $.57 * *$ & $.23 * *$ & $.50 * *$ & $.00 * *$ \\
\hline Picture naming & .98 & .04 & & $.13^{* *}$ & $.50 * *$ & $.44 * *$ \\
\hline \multicolumn{7}{|l|}{ Other tasks } \\
\hline Auditory word-picture matching & .97 & .04 & .97 & 1.00 & .94 & 1.00 \\
\hline Auditory lexical decision & .94 & .03 & .86 & .86 & $.84^{*}$ & .94 \\
\hline Visual lexical decision & 1.00 & .01 & $.88^{*}$ & .98 & $.88^{*}$ & $.90 *$ \\
\hline Phoneme discrimination & .94 & .02 & $.68^{* *}$ & .93 & $.72 * *$ & $.40 * *$ \\
\hline
\end{tabular}

* Greater than 2 standard deviations below control subjects' mean.

** Greater than 3 standard deviations below control subjects' mean.

damage to the caudate nucleus, putamen, thalamus, and white matter of the internal capsule (MRI). L.L. is a 56-year-old female with 12 years of education who participated 7 years after a CVA. She has a left frontotemporal cortical lesion along with damage to the thalamus, putamen, and extensive white-matter damage (MRI). T.R. is a 67-year-old male with 8 years of education who participated 2 years after a CVA. G.Z. is a 69-year-old female with 12 years of education who participated 20 years after an aneurysm. The sites of brain injury were not confirmed for T.R. and G.Z. because metallic implants disqualified them from undergoing an MRI. However, lefthemisphere damage can be inferred from right hemiplegia in both cases.

Table 1 presents the patients scores on subtests of Caplan's (1992) Psycholinguistic Assessment of Language (PAL) which is a psycholinguistically oriented aphasia battery. Control data were obtained from a sample of 25 normal subjects between the ages of 61 and 70 years (provided by D. Caplan). Tasks requiring verbal production are presented in the first three rows of Table 1: Oral Reading (of written words and pseudowords), Oral Repetition (of spoken words and pseudowords), and Picture Naming. It is clear that each of the four patients exhibits a drastic impairment on tasks requiring verbal production of words. Patients performed better on tasks that did not require verbal production. All patients were able to match auditory words with their corresponding pictures. Patients experienced some difficulties with the auditory and visual lexical decision tasks, but any impairment was minor compared to their verbal production problems. Finally, three of four patients showed substantial impairments on the phoneme discrimination task. The latter impairment may be attributable to hearing deficits. However, more accurate performance on auditory word-picture matching and auditory lexical decision suggest that their ability to hear words was intact, which is most critical for the present experiment in which all stimuli were words. 
Two controls were matched to each patient, on the basis of sex, age, and years of education. We were unable to precisely match years of education for R.C. and T.R. who only had 6 and 8 years, respectively. Both of R.C.'s controls had 12 years of education. One of T.R.'s controls had 8 years, but the other had 12 years of education. Controls were recruited through Massachusetts General Hospital and Harvard University and they were paid \$7 to $\$ 10$ per hour for participation.

Design, materials, and apparatus. The independent variables were group (patients vs controls) and the within-subject variable of study condition (liking ratings, ascender/descender counting, and nonstudied). Participants completed one study-test block. The study list consisted of 24 experimental words surrounded by 8 -word primacy and recency buffers. Participants rated the pleasantness of half the words and counted ascending and descending letters for the others. Words in each study condition were randomly intermixed within a single list. The test list contained 48 three-letter word stems (e.g., TRU__ ). Half of the stems could be completed with a studied word, and the other half could not be completed with a studied word.

The experimental stimuli were 96 common English words that each began with a different three-letter combination. For counterbalancing purposes, the words were subdivided into eight 12-word subsets (A1, A2, A3, A4, B1, B2, B3, B4). Each subset was roughly balanced according to word frequency $(\mathrm{MN}=12.25, \mathrm{SD}=8.74$, Range $=2$ to 40, Kucera \& Francis, 1967), word length $(\mathrm{MN}=6.23, \mathrm{SD}=.81$, Range $=5$ to 9$)$, possible number of completions with Kucera and Francis frequency greater than zero $(\mathrm{MN}=$ $15.46, \mathrm{SD}=11.41$, Range $=3$ to 59 ), and the rank frequency of the word among all alternative completions with frequency greater than zero $(\mathrm{MN}=$ 6.02 , $\mathrm{SD}=3.65$, Range $=1$ to 19 ). An additional 16 words with similar characteristics were used as buffer items. The design did not ensure that each item appeared equally often in each condition, but more importantly, patients and matched controls received the same items in the same conditions, so group differences are unlikely to be attributable to item effects. ${ }^{1}$

Stimuli were presented on a Macintosh Powerbook in lowercase, 36-point Geneva font. Responses were either written or spoken aloud depending on the subject's capability.

Procedure. During the study list, the participants either rated how much they liked each word on a scale from 1 to 7 (1 for extremely disliked words and 7 for extremely liked words) or they counted the number of letters in the word which are ascenders and descenders. Ascenders are letters which extend above the main body of the word (e.g., t, i, f) and descenders are letters which extend below (e.g., y, p, g). Each trial started with the presentation of the encoding task ("Ascender/Descenders" or "Liking") on the bot-

${ }^{1}$ Though items were selected from a pool of 96 words, each participant was presented with only 48 words in this experiment. 
TABLE 2

Mean Proportion of Stems Completed with Target Words for Experiments 1, 2, and 3.

\begin{tabular}{|c|c|c|c|c|c|c|c|}
\hline \multirow[b]{2}{*}{ Experiment } & \multirow[b]{2}{*}{ Presentation } & \multirow[b]{2}{*}{ Instructions } & \multirow[b]{2}{*}{ Condition } & \multicolumn{2}{|c|}{ Controls } & \multicolumn{2}{|c|}{ Patients } \\
\hline & & & & Means & $\begin{array}{l}\text { Old- } \\
\text { New }\end{array}$ & Means & $\begin{array}{l}\text { Old- } \\
\text { New }\end{array}$ \\
\hline \multirow[t]{3}{*}{1} & \multirow[t]{3}{*}{ Visual } & \multirow[t]{3}{*}{ Implicit } & Liking & .21 & .05 & .22 & .12 \\
\hline & & & Letters & .26 & .10 & .29 & .19 \\
\hline & & & New & .16 & & .10 & \\
\hline \multirow[t]{3}{*}{2} & \multirow[t]{3}{*}{ Auditory } & \multirow[t]{3}{*}{ Implicit } & Liking & .27 & .13 & .15 & .04 \\
\hline & & & Clarity & .27 & .13 & .14 & .03 \\
\hline & & & New & .14 & & .11 & \\
\hline \multirow[t]{3}{*}{3} & \multirow[t]{3}{*}{ Auditory } & \multirow[t]{3}{*}{ Explicit } & Liking & .50 & .46 & .25 & .07 \\
\hline & & & Clarity & .37 & .33 & .31 & .13 \\
\hline & & & New & .04 & & .18 & \\
\hline
\end{tabular}

tom of the screen. The experimenter would then initiate presentation of the word in the center of the screen, and the word would remain on the screen until the participant gave a response. The study list was followed by the distractor task of generating names of U.S. Presidents for 5 min.

At test, the participant was visually presented with 48 three-letter word stems. Half could be completed with studied words and half could not. Studied and nonstudied stems were randomly intermixed within the test list. Participants were asked to write down the first word that came to mind, avoiding proper nouns and avoiding words of four letters or less. Participants were not told that some of the stems could be completed with words from the previous task. The duration of each test trial was self-paced.

\section{Results}

The results of Experiment 1 suggest that both groups showed similar amounts of priming. Table 2 shows the proportion of stem completed with target words and priming scores (old-new) in each condition. $T$ tests confirmed that each group showed significant priming when target completion rates for old (studied) items (collapsed across encoding conditions) were compared with target completion rates for new (not studied) items: controls, $t(3)=3.02, \mathrm{SE}=.02$; patients, $t(3)=2.96, \mathrm{SE}=.03$ (both $p<.05$, onetailed). ${ }^{2}$

Priming scores (old-new, see Table 2) also were entered into a group by encoding task ANOVA, but no effects approached significance. Although the small sample sizes in this experiment limits the power to detect small effects, the trends in the results are reassuring in two respects. First, the

${ }^{2}$ Prior to all analyses data from each pair of matched controls were averaged together to equate the degrees of freedom between patients and controls. 
patients tended to show larger priming effects than did the controls, so an inability to detect an impairment in this sample of patients is probably not attributable to low statistical power. Second, priming scores tended to be larger after the letters task compared to the liking task. Because this is the opposite of the levels-of-processing effect normally observed on explicit memory tasks, it is unlikely that explicit memory had much impact on the present results (cf. Bowers \& Schacter, 1990).

In summary, the present experiment found no differences in visual implicit memory when brain-injured patients with verbal production deficits were compared with a control group. We discuss these results further after Experiment 2, in which we examine cross-modal priming in the same patients on the stem completion task used in Experiment 1.

\section{EXPERIMENT 2}

\section{Method}

Participants. The same four patients described in Experiment 1 participated in Experiment 2. Matched controls (two per patient) were selected according to the same criteria as in Experiment 1. Three of the eight controls previously participated in Experiment 1 . From 1 to 7 months intervened between Experiments 1 and 2 for subjects who participated in both.

Design, materials, and apparatus. The design was similar to Experiment 1 except that words were heard rather than seen during the study list, and the perceptual encoding task was modified accordingly. Subjects completed one study-test block. The study list consisted of 40 experimental words surrounded by 5 -word primacy and recency buffers. Subjects rated the pleasantness of half the words and rated the clarity of pronunciation for the other half. Words in each study condition were randomly intermixed within a single list. Test lists contained 80 three-letter word stems (e.g., TRU_ ). Half of the stems could be completed with a studied word, and the other half could not be completed with a studied word.

The experimental stimuli were 80 common English words that each began with a different three-letter combination. For counterbalancing purposes, the words were subdivided into four 20-word subsets. Each subset was roughly balanced according to word frequency $(\mathrm{MN}=53.34, \mathrm{SD}=48.06$, Range $=1$ to 282 , Kucera \& Francis, 1967), word length $(\mathrm{MN}=7.64, \mathrm{SD}=2.07$, Range $=5$ to 13 ), possible number of completions with Kucera and Francis frequency greater than zero $(\mathrm{MN}=18.58, \mathrm{SD}=16.69$, Range $=1$ to 72$)$, and the rank frequency of the word among all alternative completions with frequency greater than zero $(\mathrm{MN}=4.97, \mathrm{SD}=6.83$, Range $=1$ to 43 ). An additional 10 words with similar characteristics were used as buffer items. Sublists were incompletely rotated through the three conditions (New, Clarity, Liking) across participants, but matched controls received the same lists. 
Study words were recorded by a single female voice and presented on a Macintosh Powerbook. Responses were either written or spoken aloud depending on the subject's capability.

Procedure. During the study list, participants either rated the clarity of the word on a scale from 1 to 5 ( 1 being the least clear and 5 being the most clear) or they rated how much they like the meaning of the word. Liking ratings used the same scale, with 1 indicate strong disliking and 5 indicating strong liking of the word. As in Experiment 1, the encoding task was shown on the computer screen prior to presentation of each study word. The study list was followed by 5-min distractor task of generating names of cities or states from three-letter stems.

At test, participants were presented with 80 three-letter visual word stems derived from the beginnings of half studied and half nonstudied words. Participants were asked to write down the first word that came to mind, avoiding proper nouns and avoiding words of four letters or less. The stem presentations in the test phase were self-paced.

\section{Results}

The results of Experiment 2 (see Table 2) indicate less priming by patients than controls. $T$ tests comparing target completion rates for studied versus nonstudied words showed that overall priming was significant for controls, $t(3)=4.48, \mathrm{SE}=.03, p<.01$ (one-tailed), but not for patients, $t(3)=$ $1.42, \mathrm{SE}=.03$. Although our ability to detect priming effects is limited by the small sample size, a significant effect was detected for the control subjects but not for the patients despite the fact that the degrees of freedom and standard errors were the same.

A group by encoding task ANOVA showed a marginally significant priming difference between groups, $F(1,6)=5.25, p=.06$. Again, although this analysis is compromised by low statistical power, it should be noted that mean priming effect for controls (.14) was numerically more than three times the size of those for patients (.04). As in Experiment 1, there is little indication of a levels-of-processing effect, so these results are unlikely to be influenced appreciably by explicit memory.

\section{EXPERIMENT 3}

Experiments 1 and 2 suggest that verbal production deficits impair crossmodal priming, but spare within-modal visual priming. These results are consistent with the hypothesis that cross-modal priming depends on recoding processes that require normal verbal production abilities. Alternatively, it has been suggested cross-modal priming may be attributable to the influence of explicit memory processes (e.g., Jacoby, Toth \& Yonelinas, 1993; Köhler et al., 1997). If cross-modal explicit memory is impaired in the patient group compared to the control group, such impairment could play a role in the 
reduced cross-modal priming observed in Experiment 2. Accordingly, explicit memory was examined in Experiment 3.

\section{Method}

Participants. The same four patients described in Experiment 1 participated in Experiment 3. Matched controls (two per patient) were selected according to the same criteria used in Experiment 1. Two of the eight controls previously participated in Experiments 1 and 2. T.R.'s education-matched control (8 years) was unavailable for Experiment 3, so an age-matched control with 12 years of education was substituted. Participants completed Experiment 3 approximately 2 years after Experiment 2.

Design, procedure, and materials. Participants heard 36 words on the study list surrounded by four-word primacy and recency buffers. Participants rated the pleasantness of half the words, and rated the clarity of pronunciation for the other half. The test list contained 72, three-letter word stems (e.g., TRU__ ). Half of the stems could be completed with a studied word, and the other half could not be completed with a studied word. Participants were instructed to complete stems only with words from the previous study list, and they were told to leave items blank if they could not remember an appropriate studied word. All other aspects of the design and procedure were identical to Experiment 2.

The experimental stimuli were 72 common English words that each began with a different three-letter combination. The words had the following characteristics: word frequency $(\mathrm{MN}=31.94, \mathrm{SD}=5.96$, Range $=1$ to 107 , Kucera \& Francis, 1967) and word length $(\mathrm{MN}=5.96, \mathrm{SD}=0.93$, Range $=5$ to 8 ). An additional 8 words with similar characteristics were used as buffer items. Items were counterbalanced as in Experiment 2.

\section{Results}

Participants in the control group showed higher levels of explicit memory than did the patients, and the patients failed to show the typical levels-ofprocessing advantage when the liking and clarity tasks are compared (Table 2 ). The dependent variable of primary interest is the completion rate difference between old and new items. An ANOVA on these difference scores yielded a main effect of group, $F(1,6)=15.31$, MSE $=.02, p<.01$, and a marginally significant group by study task interaction, $F(1,6)=3.96$, $\mathrm{MSE}=.01, p<.10$. Furthermore, target complete rates in the new condition were higher for patients than for controls, $t(6)=2.87, \mathrm{SE}=.05, p<.05$.

\section{GENERAL DISCUSSION}

The present experiments investigated modality effects on visual stem completion in a group of brain-injured patients with verbal production deficits. 
When words were studied visually (Experiment 1), similar levels of priming were observed in the patients and the control group. When words were studied auditorily (Experiment 2), only the control group showed significant levels of priming. When explicit stem completion was tested after auditory study (Experiment 3), memory was more accurate for controls than for patients.

The results of Experiments 1 and 2 are consistent with the idea that crossmodal priming depends on recoding the perceptual format of stimuli (e.g., Downes et al., 1996; Kirsner et al., 1989; McClelland \& Pring, 1991), because our patients are impaired in their ability to produce the phonological form of visually presented stimuli. More specifically, cross-modal priming in Experiment 2 may have depended upon phonological recoding of the visual stem during the test. This recoding would allow for an auditory/ phonological match between the auditorally presented words and the visually presented stem.

Although our results are generally consistent with the idea that crossmodal priming depends on verbal recoding of visual stems, some aspects of our results are inconsistent with particular versions of this hypothesis. Kirsner et al. (1989) suggested that overall priming effects can be decomposed into two components. A perceptually specific component $\left(P_{\mathrm{s}}\right)$ depends on a match between the modality of the study and test items. The nonspecific component $\left(P_{\mathrm{ns}}\right)$ depends on verbal production or "preproduction" (meaning nonovert) processes. According to Kirsner et al., both of these components will additively contribute to within-modal priming $\left(P_{\mathrm{s}}+P_{\mathrm{ns}}\right)$, but only the nonspecific $\left(P_{\mathrm{ns}}\right)$ component will contribute to cross-modal priming. According to this model, the present patients would have a smaller nonspecific component than the control subjects $\left(P_{\mathrm{ns}}(\right.$ control $)>P_{\mathrm{ns}}($ patient $\left.)\right)$. Because the nonspecific component $\left(P_{\mathrm{ns}}\right)$ also contributes to within-modal priming $\left(P_{\mathrm{s}}+P_{\mathrm{ns}}\right)$, this model would predict greater within-modal priming in the controls subjects: If $P_{\mathrm{ns}}($ control $)>P_{\mathrm{ns}}($ patient $)$ and $P_{\mathrm{s}}($ control $)=P_{\mathrm{s}}$ (patient), then $P_{\mathrm{ns}}($ control $)+P_{\mathrm{s}}($ control $)>P_{\mathrm{ns}}($ patient $)+P_{\mathrm{s}}($ patient $)$. Therefore, the present results are inconsistent with the additivity of $P_{\mathrm{ns}}$ and $P_{\mathrm{s}}$ in withinmodal conditions. Downes et al. (1996) similarly suggest that phonological processes contribute to within- and cross-modal priming. The present results would be more consistent with a model in which verbal production response only contribute to cross-modal priming.

The aforementioned theories explain the finding of greater within- than cross-modal priming by positing that a perceptually specific contribution to priming is only available for within-modal priming. With this in mind, it is important to note that no modality effect was shown by the control group in the present experiments. Controls showed similar levels of priming when study was visual (Experiment 1) and auditory (Experiment 2). One likely explanation for the lack of a between-experiment modality effect is the use of different items in the two experiments. However, items were held constant between patients and controls, so item differences cannot explain our primary 
finding of normal within-modal priming and impaired cross-modal priming in patients with verbal production deficits.

Alternative explanations for the basis of cross-modal priming must be considered because of the explicit memory deficits patients exhibited in Experiment 3. First, "true"' priming effects might be modality specific, with crossmodal priming attributable to contamination from explicit memory processes (e.g., Jacoby et al., 1993; Kohler et al., 1997). Explicit contamination would readily explain the cooccurrence of explicit memory and cross-modal priming deficits along with normal within-modal priming in the present patients. However, explicit contamination cannot explain reports of spared crossmodal priming in some amnesic patients with explicit memory deficits (Carlesimo, Fadda, Sabbadini \& Caltagirone, 1994; Graf et al., 1985). Second, patients' explicit memory did not benefit from semantic encoding, so it is possible that they have subtle semantic encoding deficits that were not detected by our screening tasks. ${ }^{3}$ If cross-modal priming is at least in part semantically based (e.g., Bassili et al., 1989), a common semantic deficit would explain the present results, but other research has shown that cross-modal priming does not vary with the level of semantic encoding (Craik, Moscovitch \& McDowd, 1994).

In contrast to the possibility that the patients' cross-modal priming deficit in a consequence of poor explicit memory, it is also conceivable that priming deficits contributed to the poor performance on the explicit test. If priming processes contribute to performance on nominally explicit tasks (Jacoby et al., 1993; Toth, Reingold \& Jacoby, 1994), then priming deficits could explain impaired performance on both the implicit and the explicit tasks.

The explicit memory deficit shown by these patients makes it difficult to offer an unambiguous interpretation of our results, but the complete pattern of results is nonetheless intriguing insofar as it contrasts with the memory capabilities of other brain-injured patients. The present patients showed spared within-modal priming, together with deficits in cross-modal priming and explicit memory. A similar pattern-spared within-modal priming, impaired cross-modal priming and explicit memory — has been reported for visual fragment completion (e.g., E_EP_A_T is a fragment for ELEPHANT) in the severely amnesic patient K.C. (Kohler et al., 1997). K.C. has lefthemisphere cortical damage that may overlap with areas damaged in the present patients as well as medial temporal lobe damage commonly observed in patients with amnesia. By contrast, other patients with anterograde amnesia show spared within- and cross-modal priming (Carlesimo et al., 1994; Graf et al., 1985a). Another contrasting pattern is observed in patients with

\footnotetext{
${ }^{3}$ The patients' high completion rates for new stems also suggests the possibility of deficits beyond verbal production processes. Participants were explicitly instructed to respond only with studied words, so the patients may have had some difficulty comprehending and/or following instructions.
} 
occipital lesions who show normal explicit memory but fail to show the usual priming advantage for within-modal priming over cross-modal priming (Gabrieli, Fleischman, Keane, Reminger \& Morrell, 1995; Keane, Gabrieli, Mapstone, Johnson \& Corkin, 1995). Considering these different patterns together, it appears that within-modal priming can be dissociated from explicit memory, but cross-modal priming and explicit memory may share processes. Future research is needed to better understand the relationship between these memory processes.

\section{REFERENCES}

Bassili, J. N., Smith, M. C., \& MacLeod, C. M. 1989. Auditory and visual word-stem completion: Separating data-driven and conceptually driven processes. Quarterly Journal of Experimental Psychology, 41A, 439-453.

Bower, G. H. 1996. Reactivating a reactivation theory of implicit memory. Consciousness and Cognition, 5, 27-72.

Bowers, J. S. (1990). Implicit memory and test awareness. Journal of Experimental Psychology: Learning, Memory, and Cognition, 16, 404-416.

Brown, A. S., \& Mitchell, D. B. 1994. A reevaluation of semantic versus nonsemantic processing in implicit memory. Memory \& Cognition, 22, 533-541.

Caplan, D. 1992. Language: Structure, processing, and disorders. Cambridge: MIT Press.

Carlesimo, G. A., Fadda, L., Sabbadini, M., \& Caltagirone, C. 1994. Visual repetition priming for words relies on the access to the visual input lexicon: Evidence from a dyslexic patient. Neuropsychologia, 32, 1089-1100.

Challis, B. H., \& Brodbeck, D. R. 1992. Levels of processing affects priming in word fragment completion. Journal of Experimental Psychology: Learning, Memory, and Cognition, 18, 595-607.

Craik, F. I. M., Moscovitch, M., \& McDowd, J. M. 1994. Contribution of surface and conceptual information to performance on implicit and explicit memory tasks. Journal of Experimental Psychology: Learning, Memory, and Cognition, 20, 864-875.

Curran, T., \& Schacter, D. L. 1996. Implicit memory and perceptual brain mechanisms. In D. Herrmann, C. McEvoy, C. Hertzog, P. Hertel \& M. Johnson (Eds.). Basic and applied memory research: Theory in context (Vol. 1, pp. 221-240). Hillsdale, NJ: Erlbaum.

Downes, J. J., Davis, E. J., De Mornay Davies, P., Perfect, T. J., Wilson, K., Mayes, A. R., \& Sagar, H. J. 1996. Stem-completion priming in Alzheimer's disease: The importance of target word articulation. Neuropsychologia, 34, 63-75.

Ellis, A. W., \& Young, A. W. 1988. Human cognitive neuropsychology. London: Erlbaum.

Gabrieli, J. D. E., Fleischman, D. A., Keane, M. M., Reminger, S. L., \& Morrell, F. 1995. Double dissociation between memory systems underlying explicit and implicit memory in the human brain. Psychological Science, 6, 76-82.

Graf, P., Shimamura, A. P., \& Squire, L. R. (1985). Priming across the modalities and priming across category levels: Extending the domain of preserved function in amnesia. Journal of Experimental Psychology: Learning, Memory, and Cognition, 11, 386-396.

Hillis, A. E., \& Caramazza, A. 1995. Converging evidence for the interaction of semantic and sublexical phonological information in accessing lexical representations for spoken output. Cognitive Neuropsychology, 12, 187-227.

Jackson, A., \& Morton, J. 1984. Facilitation of auditory word recognition. Memory \& Cognition, 12, 358-574. 
Jacoby, L. L., \& Dallas, M. 1981. On the relationship between autobiographical memory and perceptual learning. Journal of Experimental Psychology: General, 110, 306-340.

Jacoby, L. L., Toth, J. P., \& Yonelinas, A. P. 1993. Separating conscious and unconscious influences of memory: Measuring recollection. Journal of Experimental Psychology: General, 122, 139-154.

Keane, M. M., Gabrieli, J. D. E., Fennema, A. C., Growdon, J. H., \& Corkin, S. 1991. Evidence for a dissociation between perceptual and conceptual priming in Alzheimer's disease. Behavioral Neuroscience, 105, 326-342.

Keane, M. M., Gabrieli, J. D. E., Mapstone, H. C., Johnson, K. A., \& Corkin, S. 1995. Double dissociation of memory capacities after bilateral occipital-lobe or medial temporal-lobe lesions. Brain, 118, 1129-1148.

Kirsner, K., Dunn, J. C., \& Standen, P. 1989. Domain-specific resources in word recognition. In S. Lewandowsky, J. C. Dunn \& K. Kirsner (Eds.). Implicit memory: Theoretical issues (pp. 99-122). Hillsdale, NJ: Erlbaum.

Köhler, S., Habib, R., Black, S. E., Szekely, C., Sinden, M., \& Tulving, E. (1997). Crossmodal priming in the densely amnesic subject K. C. Brain \& Cognition, 35, 420-423.

Kucera, H., \& Francis, W. N. 1967. Computational analysis of present-day American English. Providence, RI: Brown Univ. Press.

McClelland, A. G. R., \& Pring, L. 1991. An investigation of cross-modality effects in implicit and explicit memory. Quarterly Journal of Experimental Psychology, 43A, 19-33.

Rajaram, S., \& Roediger, H. L. 1993. Direct comparison of four implicit memory tests. Journal of Experimental Psychology: Learning, Memory, and Cognition, 19, 765-776.

Ratcliff, R., \& McKoon, G. 1996. Bias effects in implicit memory. Journal of Experimental Psychology: General, 125, 403-421.

Roediger, H. L. 1990. Implicit memory: Retention without remembering. American Psychologist, 45, 1043-1056.

Roediger, H. L., \& Blaxton, T. A. 1987. Effects of varying modality, surface features, and retention interval on priming in word fragment completion. Memory and Cognition, 15, 379-388.

Roediger, H. L., Weldon, M. S., Stadler, M. A., \& Riegler, G. L. 1992. Direct comparison of two implicit memory tests: Word fragment and word stem completion. Journal of Experimental Psychology: Learning, Memory, and Cognition, 18, 1251-1269.

Roediger, H. L. I., \& McDermott, K. B. 1993. Implicit memory in normal human subjects. In H. Spinnler \& F. Boller (Eds.). Handbook of neuropsychology (Vol. 8, pp. 63-131). Amsterdam: Elsevier.

Schacter, D. L. 1987. Implicit memory: History and current status. Journal of Experimental Psychology: Learning, Memory, and Cognition, 13, 501-518.

Schacter, D. L. 1990. Perceptual representation systems and implicit memory: Toward a resolution of the multiple memory system debate. In A. Diamond (Ed.). The development and neural bases of higher cognitive functions (Vol. 608, pp. 543-571). New York: Annals of the New York Academy of Sciences.

Schacter, D. L. 1994. Priming and multiple memory systems: Perceptual mechanisms of implicit memory. In D. L. Schacter \& E. Tulving (Eds.). Memory systems 1994. Cambridge, MA: MIT Press.

Schacter, D. L., \& Buckner, R. L. (1998). Priming and the brain. Neuron, 20, 185-195.

Schacter, D. L., \& Church, B. A. 1992. Auditory priming: Implicit and explicit memory for words and voices. Journal of Experimental Psychology: Learning, Memory, and Cognition, 18, 915-930. 
Schacter, D. L., \& Graf, P. 1989. Modality specificity of implicit memory for new associations. Journal of Experimental Psychology: Learning, Memory, and Cognition, 15, 3-12.

Squire, L. R. 1994. Declarative and nondeclarative memory: Multiple brain systems supporting learning and memory. In D. L. Schacter \& E. Tulving (Eds.). Memory Systems 1994. Cambridge, MA: MIT Press.

Thapar, A., \& Greene, R. L. 1994. Effects of level of processing on implicit and explicit tasks. Journal of Experimental Psychology: Learning, Memory, and Cognition, 20, 671-679.

Toth, J. P., Reingold, E. M., \& Jacoby, L. L. 1994. Toward a redefinition of implicit memory: Process dissociations following elaborative processing and self-generation. Journal of Experimental Psychology: Learning, Memory, and Cognition, 20, 290-303.

Tulving, E., \& Schacter, D. L. 1990. Priming and human memory systems. Science, 247, 301305. 